\title{
Digestion of Raw and Roasted Almonds in Simulated Gastric Environment
}

\author{
Fanbin Kong $\cdot$ R. Paul Singh
}

Received: 20 June 2009 / Accepted: 28 September 2009 /Published online: 22 October 2009

(C) The Author(s) 2009. This article is published with open access at Springerlink.com

\begin{abstract}
Knowledge of digestion kinetics of solid foods in human stomach, as affected by food processing methods, is critical in establishing processing conditions at the manufacturing stage to achieve desirable release of nutrients in the gastrointestinal tract. The objective of this study was to investigate how roasting affected disintegration and solid release properties of almond in simulated gastric environment. In vitro trials were performed for raw and roasted almonds by using static soaking method and a model stomach system. The changes in sample weight, dry mass, and moisture during the trials were determined. Both compression and penetration tests were used to investigate the texture of almonds with a focus on the influence of absorption of gastric juice. Light microscopy and transmission electronic microscopy were used to study the change in microstructure of the raw and roasted almonds after simulated digestion. The results suggested that the slow disintegration rate and the high amount of swelling of the almonds in the stomach may contribute to their high satiety property. Roasting significantly improved the disintegration rates of almonds and increased loss of solids during simulated digestion, which is well correlated with the decrease in the rigidity of almond samples after absorbing gastric juice. Microstructure of digested almonds showed breakage and breach of cell walls due to acid hydrolysis. Intercellular and intracellular channels formed in almonds
\end{abstract}

F. Kong $\cdot$ R. P. Singh $(\bowtie)$

Department of Biological and Agricultural Engineering,

University of California,

Davis, CA 95616, USA

e-mail: rpsingh@ucdavis.edu

R. P. Singh

Riddet Institute, Massey University,

Palmerston North, New Zealand during roasting are important for penetration of gastric juice that may facilitate an effective digestion.

Keywords Almond - Roasting · Digestion · Disintegration . Stomach $\cdot$ Texture $\cdot$ Microstructure

\section{Introduction}

Food processing has evolved to a step to create and design food structures to affect food bioaccessibility and bioavailability and to control the release of nutrients at the desired site and rate in the human gastrointestinal (GI) tract. This requires a good understanding on how food processing affects structure and texture and its influence on digestion properties. Also, it is important to understand the changes that occur in foods in the human GI tract leading to the breakdown of food matrix and release of food nutrients. Currently, available information in the literature is limited on the digestion kinetics of foods and its relationship with material properties such as texture and microstructure. In this paper, we studied how roasting affected almond disintegration and the release of solid matter in a simulated gastric environment. The results were related with the changes in texture and microstructure of raw and roasted almonds during digestion.

The consumption of tree nuts has increased by $45 \%$ in the United States over the last decade, and almond (Prunus dulcis) consumption has increased the most. ${ }^{1}$ California is the major grower of almonds in the US, and more than half of the state's production is the "Nonpareil" variety. Almonds contain $\sim 21 \%(w / w)$ protein and $\sim 50 \%(w / w)$ oil content in which oleic and linoleic acids are the major constituents. Almonds are also a nutrient-dense source of vitamin E, manganese, magnesium, copper, phosphorus, fiber, and 
riboflavin. ${ }^{2}$ Studies have shown that almonds have beneficial effects on cardiovascular health, obesity-related diseases, and certain cancers. ${ }^{2,3}$ Roasted almonds are a popular product consumed as a snack food. It is also used as an ingredient in many bakery and confectionary foods, such as sweets, chocolate, and biscuits. Roasting enhances the flavor and significantly alters the color and texture. The conditions of roasting generally used by processors are heating to 100 $200^{\circ} \mathrm{C}$ and a residence time of 10 to $60 \mathrm{~min}^{4,5}$

Almonds possess rigid texture that may make it hard to breakdown in the stomach during digestion. Ellis and others reported that a significant proportion of raw almond tissue is preserved intact even after chewing, digestion, and large bowel fermentation. ${ }^{6}$ On the other hand, food processing such as boiling significantly changes the food texture, thus modifying the rate of gastric disintegration. ${ }^{7}$ To our knowledge, no study has been conducted on the rate of almond disintegration in gastric environment as related to the roasting conditions, although in vitro gastric digestion was used to evaluate the nutrient bioaccessibility including protein, lipid, and vitamin E of almonds. ${ }^{8}$ Research is also required to achieve an improved understanding of the role that the texture and microstructure of almonds and their inherent changes during digestion play on disintegration kinetics.

In vivo approaches such as feeding study, intubation techniques, scintigraphy, ultrasonography, and magnetic resonance imaging have been used to assess gastric disintegration and emptying of food and drugs in clinical and research settings. Besides, a number of in vitro GI tract models, such as the TNO intestinal model, are currently available for nutrition, toxicology, pharmacology, and safety assessments. ${ }^{9}$ The advantages of in vitro techniques include no ethical constraints, reduced cost, and improved accuracy and reproducibility. In our previous paper, we have presented an in vitro model stomach system to investigate disintegration kinetics of solid foods in human stomach. ${ }^{7}$ This model is useful in studying the disintegration and release of solid matter from different foods as it provides adjustable and measurable hydrodynamic and/or mechanical forces. The aim of the current study was to evaluate the disintegration and solid release characteristics of raw and roasted almonds using a static soaking method and the model stomach system and to investigate the relationship between the digestion properties of almonds and their texture and microstructure.

\section{Materials and Methods}

Preparation of Samples

Shelled raw almonds of the Nonpareil variety (Diamond Foods, Stockton, CA, USA) were purchased in a grocery store. All samples were stored at $3-5^{\circ} \mathrm{C}$. Based on a random sampling of ten almonds, the following measurements were obtained: weight $(1.42 \pm 0.04 \mathrm{~g})$, length $(2.39 \pm$ $0.08 \mathrm{~cm})$, width $(1.42 \pm 0.05 \mathrm{~cm})$, and thickness $(0.86 \pm$ $0.04 \mathrm{~cm}$ ). Almonds were roasted in a household toaster oven (Hamilton Beach Brands, Washington, NC, USA) at $185^{\circ} \mathrm{C}$ for 9 min. In vivo studies have shown that, after chewing, the size of masticated almond particulates is mostly less than 3 to $4 \mathrm{~mm} .{ }^{16}$ To facilitate convenient sample loading and achieve a substantial percentage mass loss in the experiment, larger size particulates $(6 \mathrm{~mm}$ in diameter and length) were used for this study. For disintegration and solid release trials, rectangular prismatic sections (length $\times$ width $\times$ height $=6 \times 6 \times 4 \mathrm{~mm}$, weight $0.15 \mathrm{~g}$ ) were cut from raw and roasted almonds. Samples with other dimensions were also prepared to investigate how particulate size influences disintegration rates, including $5 \times 5 \times 4,4 \times 4 \times 4$, and $3 \times 3 \times 3 \mathrm{~mm}$, with a weight of $0.10,0.06$, and $0.03 \mathrm{~g}$, respectively.

Simulated gastric juice and saliva were prepared as described in a previous paper. ${ }^{7}$ Simulated gastric juice $(\mathrm{pH}$ 1.8) comprised of pepsin $(1 \mathrm{~g} / \mathrm{L})$, gastric mucin $(1.5 \mathrm{~g} / \mathrm{L})$, and $\mathrm{NaCl}(8.775 \mathrm{~g} / \mathrm{L})$. Simulated saliva included gastric mucin $(1 \mathrm{~g} / \mathrm{L}), \alpha$-amylase $(2 \mathrm{~g} / \mathrm{L}), \mathrm{NaCl}(0.117 \mathrm{~g} / \mathrm{L}), \mathrm{KCl}$ $(0.149 \mathrm{~g} / \mathrm{L})$, and $\mathrm{NaHCO}_{3}(2.1 \mathrm{~g} / \mathrm{L})$. All chemicals were purchased from Sigma-Aldrich (USA).

\section{Static Soaking Test}

The samples $(6 \times 6 \times 4 \mathrm{~mm})$ were first soaked in the simulated saliva at $37^{\circ} \mathrm{C}$ for $15 \mathrm{~s}$ to incorporate the effect of oral exposure on food digestion. The samples were withdrawn from the simulated saliva and blotted to remove excess water. The weights were measured as initial weight $W_{0}$ (in grams). Then samples were soaked in gastric juice in a $37^{\circ} \mathrm{C}$ water bath for different time periods, from 1 to $5 \mathrm{~h}$, as most foods empty from stomach to intestine within 5 h. ${ }^{9}$

Each particulate sample was hooked into the tip of a rigid steel wire and suspended in $20 \mathrm{~mL}$ gastric juice in a test tube. The sample was suspended in the central part of the tube. After soaking, the weight of the sample $W_{t}$ (in grams) was measured again. The moisture of the sample was determined by using a hot air oven at $105^{\circ} \mathrm{C}$ until constant weight was achieved (typically in $18 \mathrm{~h}$ ). $M_{0}$ (in percent) and $M_{t}$ (in percent) were denoted as initial moisture and the moisture after trial time $t$ in dry basis. Dry mass of samples was calculated based on the sample weight and moisture. Weight retention ratio and dry mass retention ratio were calculated as follows:

Weight retention ratio, $y_{t}=\frac{W_{t}}{W_{0}}$ 
where $W_{0}$ (in grams) is the initial weight and $W_{t}$ (in grams) is the sample weight after time $t$ (in minutes).

Dry mass retention ratio, $S_{t}=\frac{\mathrm{DM}_{t}}{\mathrm{DM}_{0}}$

where $\mathrm{DM}_{0}$ is the initial dry mass (in grams) and $\mathrm{DM}_{t}$ (in grams) is the dry mass after time $t$ (in minutes).

Static soaking of whole almond kernels was also conducted to evaluate the change in the density and volume of almonds during digestion. About $26 \mathrm{~g}$ ( $\sim 19$ kernels) raw and roasted almond samples (whole kernel with skin) were soaked in $100 \mathrm{~mL}$ simulated gastric juice contained in beakers covered by aluminum foil at $37^{\circ} \mathrm{C}$ water bath for $16 \mathrm{~h}$. After soaking, the samples were carefully drained and the samples were blotted using filter paper. Changes in the sample weight were determined. The kernel volume was obtained by determining the change in the volume of gastric juice with or without almond kernels.

In Vitro Digestion of Almonds in the Model Stomach System

The in vitro digestion test for raw and roasted almonds was conducted in a model stomach system as described in our previous paper. ${ }^{7}{ }^{10}$ The stomach model is comprised of a glass chamber which holds simulated gastric juice at $37^{\circ} \mathrm{C}$. The chamber is placed on a turntable and rotated at selected revolutions per minute. Almond samples were fixed in a sample holder and submerged into gastric juice at about $1.5 \mathrm{~cm}$ depth. In this study, the sample holder was connected to a reaction torque cell (Omega Engineering, TQ201-25Z) and a data acquisition system (Omega Engineering, OMB-DAQ-54) to measure forces acting on the samples during trials. The samples were tested under two different experimental settings, depending on whether or not plastic beads (GP-22 Natural ABS Plastic Beads, BASF Corporation, New Jersey) were added into the simulated gastric juice. These plastic beads, incorporated into gastric juice to mimic food particulates in human stomach, have a size of $3 \mathrm{~mm}$ diameter and specific gravity of 1.03. When plastic beads were not combined with the gastric juice, which is referred to as "trial A," samples were subjected to hydrodynamic shearing stress caused by the rotating fluid; when plastic beads were combined with the gastric juice, referred to as "trial B" or "disintegration test," a mechanical force was created resulting from the beads impacting and grinding the samples that caused food disintegration, simulating the forces due to contraction that are present in a human stomach due to peristaltic movement. ${ }^{7}$ The approach of using plastic beads to create mechanical force originated in drug dissolution studies. ${ }^{23}$ This method was adopted in our model stomach system. ${ }^{7}$
The beads effectively generated mechanical force by impacting and grinding food samples during trials. The stable physical and chemical properties of the ABS beads and their high mechanical strength to resist friction provide sustainable and repeatable force conditions.

For trial A, samples were first soaked in the simulated saliva for $15 \mathrm{~s}\left(37^{\circ} \mathrm{C}\right)$. The sample surfaces were blotted using a filter paper, and the weight was determined as the initial weight $W_{0}$ (in grams). The samples were then tested in the model stomach system. The model contained $500 \mathrm{~mL}$ of simulated gastric juice with a rotation speed set at $30 \mathrm{rpm}$. For each trial, four samples were tested simultaneously. The force measured using the torque cell was approximately $0.001 \mathrm{~N}$ for each sample. The trials were continued for $1,2,3,4$, and $5 \mathrm{~h}$, respectively. Then the model was stopped and samples were taken out, dried with a filter paper to remove surface moisture, and measured for weight $W_{t}$ (in grams). The moisture and dry mass content were subsequently determined for each sample.

For trial B, $330 \mathrm{~g}$ plastic beads were combined with $170 \mathrm{~mL}$ simulated gastric juice to make $500 \mathrm{~mL}$ simulated gastric content. This ratio of beads weight/juice volume was selected to generate the mechanical force simulating the highest level of stomach force measured in vivo as reported in literature. All trials were conducted following the same procedure as trial $\mathrm{A}$. The mechanical force generated by beads impacting on the sample was approximately $0.1 \mathrm{~N}$, which is comparable to the maximum force present in the human stomach. ${ }^{10}$ The trials were continued until 1, 2, 3, 4, and $5 \mathrm{~h}$, respectively. The weight retention, moisture, and dry mass retention ratio of samples for each trial were determined accordingly.

Trial B was also conducted to evaluate the effect of sample size on disintegration rates of raw almonds. For this experiment, samples with different dimensions were tested in the stomach model. Same samples were treated for $6 \mathrm{~h}$, and the weight changes were determined with $1 \mathrm{~h}$ time interval.

\section{Textural Measurement}

Compression test and penetration test were used to determine textural properties of raw and roasted almonds, both before and after static soaking in gastric juice.

\section{Uniaxial Compression Test}

The rectangular prismatic section samples $(6 \times 6 \times 4 \mathrm{~mm})$ before and after static soaking for $7.5 \mathrm{~h}$ were used for compression test. A cylindrical flat-end probe $(4 \mathrm{~cm}$ diameter) was fitted to the Texture Analyzer TA-XT2 (Texture Technologies, Scarsdale, NY, USA/Stable Micro Systems, Godalming, Surrey, UK) equipped with a load cell 
of $50 \mathrm{~kg}$. The probe traveled to $3 \mathrm{~mm}$ depth into the samples at a speed of $1 \mathrm{~mm} / \mathrm{s}$. Samples were compressed at a direction transversal to the sample height. During the test run, the resistance of the sample was recorded for every $0.01 \mathrm{~s}$ and plotted as a force (in newtons) vs. time (in seconds) graph. The following measurements were carried out to characterize the mechanical properties of the raw and roasted almonds before and after soaking ${ }^{11-13}$ :

(a) Fracture force, $F$ (in newtons): the force at the first major failure event.

(b) Deformation, $\delta$ (in millimeters): the absolute deformation where the first break occurred.

(c) Hardness after fracture, $H$ (in newtons): the maximum force values after fracture point, which were usually the force at $3 \mathrm{~mm}$ depth (example curves shown later in the "Results and Discussion" section).

(d) Compression work, $A$ (in newton millimeter): area under the curve, force vs. distance, in the test for compression. It is considered as an empirical index of toughness and associated with total energy input during compression.

(e) Stiffness, $S$ (in newtons per millimeter): the slope of the line joining the origin and the peak force.

\section{Penetration Test}

Rectangular prismatic section samples $(10 \times 10 \times 4 \mathrm{~mm})$ were cut from the raw and roasted whole kernel almonds both before and after soaking for $16 \mathrm{~h}$. A flat-ended needle probe ( $2 \mathrm{~mm}$ diameter) was fitted to the Texture Analyzer TA-XT2. The probe traveled to $3 \mathrm{~mm}$ depth transversal to the sample height at a speed of $1 \mathrm{~mm} / \mathrm{s}$, and the following parameters were calculated for the force-distance curves:

(a) Fracture force, $F$ (in newtons): the force at the first break of the sample.

(b) Deformation, $\delta$ (in millimeters): the deformation at the fracture point.

(c) Hardness after fracture, $H$ (in newtons): the maximum force value after the first break.

(d) Penetration work, $A$ (in newton millimeter): area under the force-distance curve.

(e) Stiffness, $S$ (in newtons per millimeter): the slope of the line joining the origin and the peak force.

\section{Microscopy Examination}

Light microscopy (LM) and transmission electron microscopy (TEM) were used to investigate changes in the microstructure of raw and roasted almonds after simulated digestion. Raw and roasted almond $\left(185^{\circ} \mathrm{C}, 9 \mathrm{~min}\right)$ samples $(5 \times 5 \times 4 \mathrm{~mm})$ were soaked in gastric juice for $60 \mathrm{~min}$ at $37^{\circ} \mathrm{C}$. LM and TEM images were obtained for the soaked raw and roasted samples following the method presented elsewhere. ${ }^{10}$

\section{Statistical Analysis}

Weight retention data ( $y_{t}$ vs. $\left.t\right)$ for the disintegration test were analyzed and fitted into empirical models. The general linear models procedure (GLM) in the SAS software package for Windows V8.01 and the Solver command in the EXCEL software were used to obtain model parameters. Estimates for parameters were obtained by minimizing the mean square error (MSE). MSE is a measure of the goodness of fit. In addition, the correlation coefficient $(r)$ was used as a criterion for evaluating the performance of the models. A significance test was conducted using analysis of variance in the GLM procedure of the SAS system. Differences between group means were analyzed by Duncan's multiple-range test. Statistical significance was set at a probability level of 0.05 .

\section{Results and Discussion}

\section{Results of Trial A and Static Soaking Test}

Figure 1 shows the changes of weight retention ratio, dry mass retention, and moisture of raw and roasted almonds with time during trial $\mathrm{A}$ in which the samples were subjected to hydrodynamic mixing when no mechanical force was involved. In the pharmaceutical industry, the United States Pharmacopeia (USP) dissolution testing is commonly used for evaluating drug tablets, capsules, and nutritional dietary supplements, which also involves mixing sample with simulated gastric juice to cause the dissolution of active ingredients. ${ }^{9}$ But the advantage of the model stomach system we used is that the hydrodynamic shearing effect on the tested samples can be controlled by adjusting the rotational speed of the turntable and the hydrodynamic forces can be measured with the torque cell simultaneously.

When a food material is mixed with gastric juice, two types of mass transfer occur, namely, diffusion of gastric juice into the food and leaching of solids (such as proteins, fibers, oils, vitamins, and minerals) from the food to the gastric fluid. The acid and enzyme in the gastric juice enhance the leaching of solids due to the hydrolysis and enzymatic reactions that reduce the cohesive forces bonding the solid material within the food matrix. The liquid concentration gradient provides the driving force for the first type of mass transfer, while the concentration difference of solids causes the driving force for the second type of mass transfer. In our study, we quantified these two processes by measuring the sample weight and moisture 

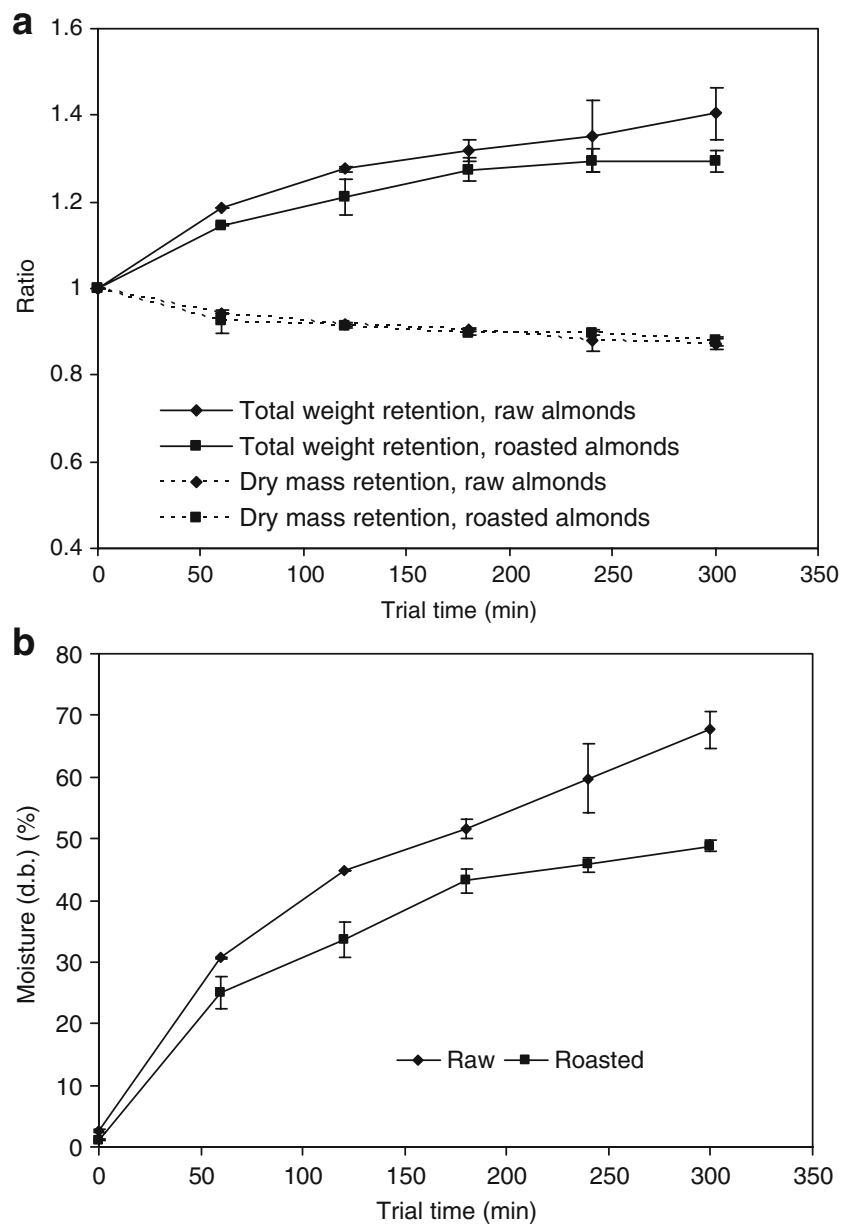

Fig. 1 Retention of total weight, dry mass, and change in moisture in raw and roasted almonds during trial A $(n=4)$. Volume of simulated gastric juice $=500 \mathrm{~mL}$; turntable rotation speed $=30 \mathrm{rpm}$

and calculated the loss of dry solids. The weight retention ratio and the dry mass retention ratio were thus calculated respectively to indicate the overall effect of food digestion and the net release of solids.

Trial A test showed that, for both raw and roasted almonds, the weight of the samples continuously increased, while the dry mass (solids) decreased (Figure 1a). There is no significant difference in the dry mass retention ratios of raw and roasted almonds, both decreased continually to approximately $87.5 \%$ after $5 \mathrm{~h}$ of treatment, corresponding to a $12.5 \%$ solids loss. The solid loss during soaking is mainly due to the loss of fat and proteins. ${ }^{8}$ The increase in sample weight was due to the absorption of gastric fluid (Figure 1b). The initial increase in water content was rapid, leading to a fast increase in sample weight within the first $2 \mathrm{~h}$. At the end of the 5-h trial, raw almonds absorbed more gastric juice than roasted almonds, $67.7 \%$ and $48.8 \%$ in moisture (d.b.), respectively, which resulted in a higher weight gain in raw almonds than roasted almonds.
To investigate how hydrodynamic mixing of gastric fluids affects the solid release of almonds, we also conducted static soaking of the raw and roasted almonds. In this case, the samples were soaked in gastric juice at $37^{\circ} \mathrm{C}$. The soaking conditions were the same as those in trial A except that there was no fluid rotation so there was no hydrodynamic shearing effect. Interestingly, the result indicated that there was no significant difference between the static soaking and trial A of the two samples (raw and roasted) in terms of weight change, moisture increase, and solid (dry mass) loss. This result indicated that, for raw and roasted almonds, when exposed to a simulated gastric environment similar to what was used here, the nutrient release will not be affected by the hydrodynamic conditions of surrounding gastric fluid. Using a computer simulation, Pal and others ${ }^{14}$ reported maximum retropulsive flow velocities in the stomach antrum of approximately $7.5 \mathrm{~mm} / \mathrm{s}$. With $30 \mathrm{rpm}$ rotational speed in our model, the flow speed impacting the samples is much higher $(204 \mathrm{~mm} / \mathrm{s})$. It is, therefore, reasonable to conclude that, in almond digestion, for leaching of nutrients, the internal resistance is more important than surface resistance.

\section{Results of Trial B}

When the mechanical force was incorporated by combining plastic beads into gastric juice in the model stomach system (trial B), the changes in weight and dry mass retention ratios and moisture for raw and roasted almonds are shown in Figure 2. The change in sample weight and dry mass is a combined effect of leaching of solids and surface erosion. Surface erosion indicates the removal of solids from the food surface by an impinging gastric fluid containing plastic beads that impact and shear the food surface. The weight retention profiles (disintegration profile) shown in Figure 2 have a delayed-exponential shape, that is, the weight retention ratio increased at first so that it was higher than 1 , then decreased gradually due to surface erosion and leaching of solids. Delayed-exponential profile resulted from the water absorption of almonds during digestion, i.e., the water absorption rate was higher than the rate of surface erosion and solid release leading to a net increase in the sample weight. ${ }^{10}$ It can be also seen that, after the same treatment, roasted almonds had more weight loss than raw almonds $(P<0.05)$. After $5 \mathrm{~h}$, the weight retention ratio was 0.93 and 0.77 for the raw and roasted almonds, respectively. In our previous paper, we proposed a linear-exponential equation to describe data obtained for the weight retention ratio vs. disintegration time ${ }^{10}$ :

$y_{t}=(1+k \cdot \beta \cdot t) \times e^{-\beta \cdot t}$

where $k$ is a dimensionless constant and $\beta(1 / \mathrm{min})$ is a parameter that measures the concavity of the time-weight 

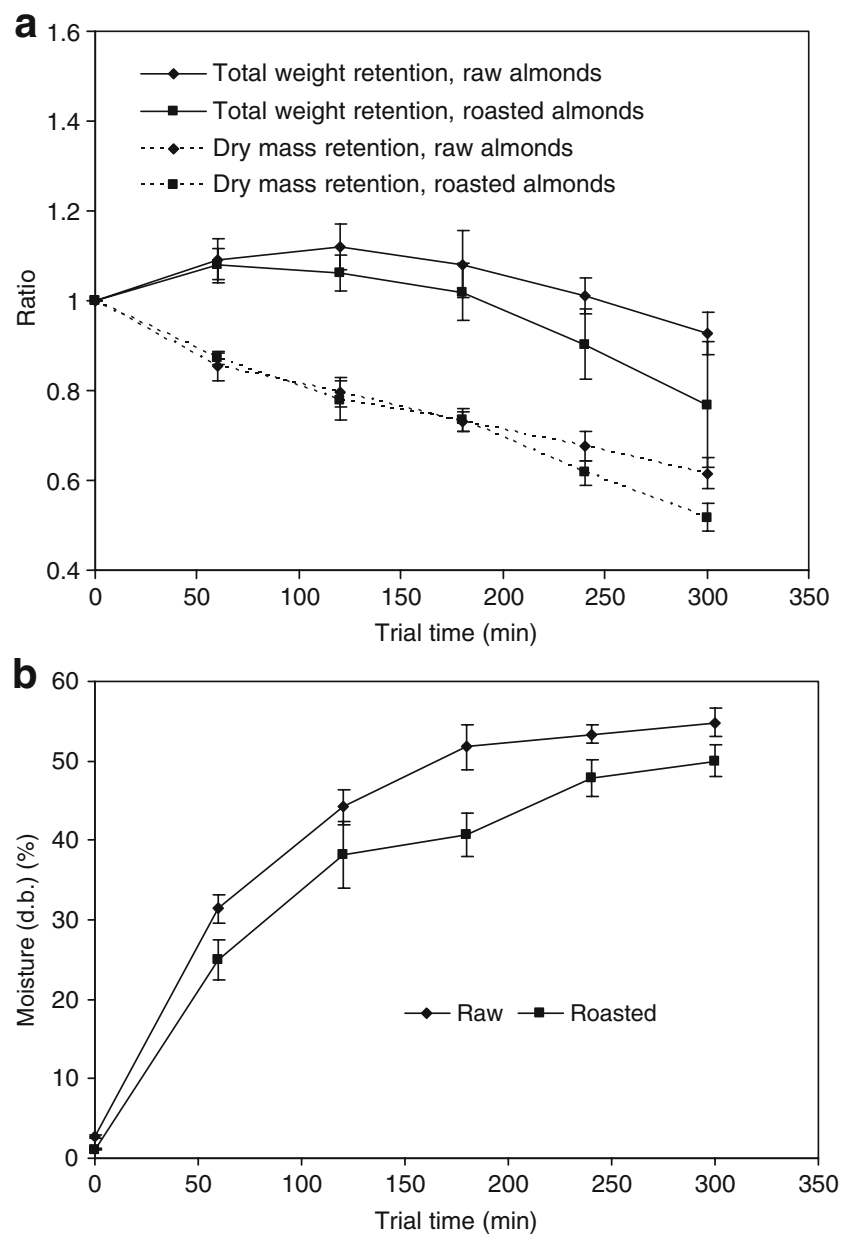

Fig. 2 Retention of total weight, dry mass, and moisture changes in raw and roasted almonds during trial $\mathrm{B}(n=4)$. Volume of simulated gastric content $=500 \mathrm{~mL}$ (gastric juice $=170 \mathrm{~mL}$, beads $=330 \mathrm{~g}$ ); turntable rotation speed $=30 \mathrm{rpm}$

retention relationship. The advantage of this equation is that it provides a good fit of three different disintegration profiles, namely, exponential, sigmoidal, and delayedsigmoidal profiles. ${ }^{10}$ The $k$ and $\beta$ values can be derived using regression. The half time (the disintegration time to achieve $50 \%$ mass loss), $t_{1 / 2}$, can be calculated by using $y_{t}=$ 0.5 . This parameter is usually used to express the disintegration rate. Table 1 shows the parameters of Eq. 3 . It shows that the $t_{1 / 2}$ for raw and roasted almond were 11 and $7.4 \mathrm{~h}$, respectively. From additional experiments, we also found that the disintegration rates increased with an increase in roasting temperature and time (data not shown).

The moisture content of samples had a rapid increase initially followed by leveling off (Figure 2b). Raw almonds had larger water absorption than roasted almonds (54.8\% vs. $50.0 \%$ ) at the end of the trial. The dry mass retention ratio continuously decreased, with the roasted sample exhibiting a slightly higher decrease (Figure 2a). After $5 \mathrm{~h}$, the dry mass retention ratio was 0.61 and 0.52 for raw and roasted almonds, respectively. These results indicate that roasting improved the disintegration of almonds and increased solid release during in vitro digestion.

The solid loss of $40-50 \%$ for almonds in trial B vs. $12.5 \%$ in trial $\mathrm{A}$ indicated that, for almonds, the mechanical forces due to peristaltic contraction of the stomach may be critical for the effective release of nutrients. This finding suggests that, when conducting in vitro testing of bioaccessibility of food/drug tablets, the mechanical force should not be neglected. The model stomach system used here is effective for studying food digestion and nutrient release as it is capable of providing measureable and controllable mechanical force to simulate in vivo contraction forces present in the stomach.

Disintegration of Raw Almonds with Different Size in Model Stomach System

As shown in the preceding sections, large size samples $(6 \times$ $6 \times 4 \mathrm{~mm}$ ) were used to compare the disintegration rates between raw and roasted samples. In reality, after mastication, almonds are broken into much smaller pieces of particulates, with only $10 \%$ larger than $2 \mathrm{~mm} .{ }^{15}$ In another study, Frecka and others ${ }^{16}$ reported that $\sim 3 \%$ of the total weight of masticated almonds have a size $>3.35 \mathrm{~mm}$. An experiment was conducted to investigate how the size of almond particulates affected the disintegration rate. Raw almond samples with different dimensions were tested in the stomach model subject to a mechanical force of $0.1 \mathrm{~N}$ for each sample (trial B). The disintegration profiles and fitted model curves (Eq. 3) are shown in Figure 3. It can be seen that all the tested samples displayed delayed-sigmoidal profiles, and the disintegration rate increased with the reducing sample size. The model parameters are shown in Table 2. The half time significantly decreased with the sample size/weight, from $10 \mathrm{~h}$ for the $6 \times 6 \times 4$-mm sample size $(0.15 \mathrm{~g})$ to $5 \mathrm{~h}$ for the $3 \times 3 \times 3-\mathrm{mm}$ sample $(0.03 \mathrm{~g})$. In this size range, a linear relationship exists between sample weight and half time:

$t_{1 / 2}=2615.4 \cdot w+214.9 \quad R^{2}=0.987$

where $w$ is the initial sample weight (in grams) ranging from 0.03 to $0.15 \mathrm{~g}$ and $t_{1 / 2}$ is the half time (in minutes).

Table 1 Model parameters of simulated gastric disintegration of raw and roasted almonds calculated with the linear exponential model (Eq. 3)

\begin{tabular}{lccccc}
\hline & $k$ & $\begin{array}{l}\beta \\
\left(\times 10^{-3} 1 / \mathrm{min}\right)\end{array}$ & $\begin{array}{l}\text { MSE } \\
\left(\times 10^{-4}\right)\end{array}$ & $r$ & $\begin{array}{l}t_{1 / 2} \\
(\mathrm{~min})\end{array}$ \\
\hline Raw & 1.664 & 3.789 & 0.11 & 1.00 & 666 \\
Roasted & 1.592 & 4.935 & 3.80 & 0.99 & 445 \\
\hline
\end{tabular}




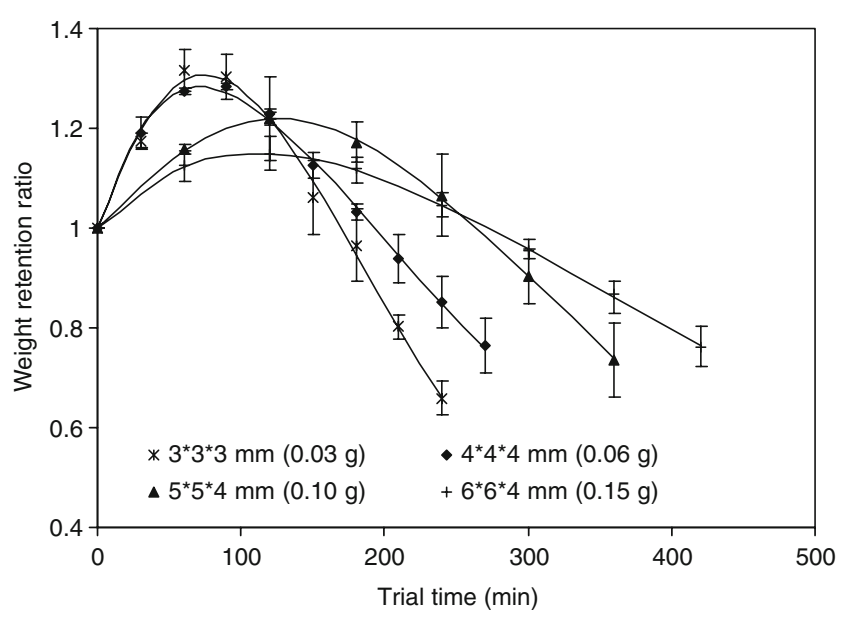

Fig. 3 Weight retention of raw almonds of different sizes fitted with Eq. $3(n=4)$. Equation parameters fitted to the data in the figure are shown in Table 2

The reduction of half time with reducing sample size emphasizes the importance of chewing. In vivo studies have shown that, during digestion in the stomach, food is reduced into 1- to 2-mm-size particulates before the chyme is discharged into the intestine, and this process usually completes within $5 \mathrm{~h}$. After $5 \mathrm{~h}$, the indigestible large objects are emptied only during phase III activity in the fasting motility pattern. ${ }^{17}$ Considering that these trials were conducted under the forces simulating the maximum mechanical force present in a human stomach, the results suggest that a considerable amount of almond particulates (both raw and roasted) may be emptied to intestine with relatively large size $(>1-2 \mathrm{~mm})$. Furthermore, the slow disintegration rate will lead to a slow stomach emptying rate and an increased feeling of fullness. Epidemiologic studies have documented that a high frequency of almond consumption may lower body mass index. ${ }^{18}$ This effect has been related with the resistance of nut parenchyma cell walls to microbial and enzyme degradation in the GI tract that results in a considerable amount of undigested almond fractions passing through the GI tract with physically intact cells. ${ }^{6,8,18}$

Water Absorption and Volume Swelling of Raw and Roasted Almonds in Gastric Environment

Static soaking tests, trial $\mathrm{A}$, and trial $\mathrm{B}$ all indicated that raw almonds absorbed more gastric juice than roasted samples during digestion. This result was further confirmed by soaking of whole kernel almonds: after $16 \mathrm{~h}$ soaking, raw and roasted almonds had $103 \%$ and $69 \%$ moisture (d. b.), compared with initial moisture of $2.7 \%$ and $1.1 \%$, respectively. This difference between the moisture of soaked raw and roasted almonds may be due to the structural damage and cell shrinkage occurring during roasting that resulted in a loss of water absorption ability in roasted almonds.

The significant water absorption in raw and roasted almonds is accompanied by volume swelling. By measuring the volume of whole almonds before and after overnight soaking, it was found that the volume increased by $44 \%$ and $32 \%$ for raw and roasted almonds, respectively. The specific gravity of raw and roasted almonds was calculated. It was initially 1.04 and 1.01 , respectively, and both decreased to approximately 1.00 after overnight soaking. Therefore, the weight retention ratio profiles as shown in Figures 1a, 2a, and 3 also reflect the approximate value of volume change during digestion. A maximum of approximately $10 \%$ increase in volume can be seen for the raw almonds with $6 \times 6 \times 4 \mathrm{~mm}$ size (Figures $2 \mathrm{a}$ and 3 ), while for smaller particulates, such as $3 \mathrm{~mm}$ cubes, the swelling can be up to $30 \%$ of original volume (Figure 3). After oral mastication, almonds are broken into even smaller pieces with only $10 \%$ larger than $2 \mathrm{~mm},{ }^{15}$ an even higher amount of swelling can be expected. We also observed that the swelling capacity increased with increasing temperature and time of roasting (data not shown). This finding may have important implications in developing healthy diets incorporating almonds. A fully chewed almond diet (or almond powder) may generate a rapid satiety feeling due to a larger increase in volume but the stomach emptying rate will be also faster, while partially chewed almonds, with the larger particulate size, may generate slower satiety feeling due to lower swelling capacity, but the fullness feeling may last for a longer time because of the slower breakdown of the large particulates. Nutrition studies have documented that nuts have high satiety properties while the mechanism remains unknown; ${ }^{18}$ our results indicated that the significant swelling and the slow disintegration rate of the ingested almonds in the stomach may be an important reason. Although no study on the swelling of almonds in gastric lumen was found in the literature, in vivo studies have shown that ingestion of dietary oat can cause stomach distension and affect satiety due to water absorption and swelling. ${ }^{19}$ Further research, especially an in vivo study, is needed to quantitatively

Table 2 Model parameters of simulated gastric disintegration of raw almonds with different dimensions calculated with the linearexponential model (Eq. 3)

\begin{tabular}{cccccc}
\hline & $k$ & $\beta$ & $\begin{array}{l}\text { MSE } \\
\left(\times 10^{-3} 1 / \mathrm{min}\right)\end{array}$ & $r$ & $\begin{array}{l}t_{1 / 2} \\
(\mathrm{~min})\end{array}$ \\
\hline $6 \times 6 \times 4 \mathrm{~mm}$ cube $(0.15 \mathrm{~g})$ & 1.78 & 3.90 & 0.09 & 1.00 & 599 \\
$5 \times 5 \times 4 \mathrm{~mm}$ cube $(0.10 \mathrm{~g})$ & 2.04 & 5.04 & 5.26 & 0.99 & 497 \\
$4 \times 4 \times 4 \mathrm{~mm}$ cube $(0.06 \mathrm{~g})$ & 2.21 & 7.29 & 1.27 & 1.00 & 357 \\
$3 \times 3 \times 3 \mathrm{~mm}$ cube $(0.03 \mathrm{~g})$ & 2.32 & 9.00 & 8.61 & 0.99 & 296 \\
\hline
\end{tabular}


characterize the swelling phenomenon of almonds in human stomach and its implications in satiety and obesity control.

Textural Changes of Raw and Roasted Almonds During Digestion

In vitro disintegration trials reported in this paper have shown that roasting can improve the disintegration rate of almonds and the solid release. It is, therefore, important to understand the underlying mechanisms that cause the observed changes in the digestion properties from the perspective of food material properties. Food texture and its change during digestion are crucial for different rates of food disintegration. ${ }^{7}, 10$ The texture of raw and roasted almonds before and after soaking in simulated gastric juice were studied with the aim to (1) investigate how almond texture and its change in the gastric environment affected its digestion performance and (2) seek textural properties that can best correlate with its digestion properties. We tried both uniaxial compression test with a flat-ended probe and penetration test using a needle probe.

\section{Uniaxial Compression Test}

Figure 4 compares the force-deformation curves for raw and roasted almonds under a compression test. Table 3 shows the values of textural parameters obtained from the curves. Significantly different textural profiles between the almonds, before and after soaking in simulated gastric juice, can be seen by comparing Figure 4a, b. Firstly, the overall resistance of almonds to compression (hardness at $3 \mathrm{~mm}$ depth and stiffness) significantly decreased after soaking, indicating a softening effect resulting from the plasticizer effect of water absorption, acid hydrolysis, and enzymatic reaction during digestion. Secondly, before soaking, the resistance increased continually during compression, so that the highest force is at the $3 \mathrm{~mm}$ depth, while after soaking, the profiles show a large decrease in the force at the fracture point, so that the highest force is also the fracture force. This indicates a more stiff texture of almonds before soaking due to their low moisture, as indicated by the higher stiffness values in the almonds before soaking.

In general, for the almonds before soaking, degree of roasting showed a limited influence on the textural properties under study, and there is no significant difference among raw and roasted almonds for the hardness, stiffness, and the total area (energy input; Table 3). However, roasted samples fractured at lower forces, and the force of the first fracture is significantly lower for the roasted almonds. Also, there are more fracture points, i.e., higher jaggedness, in the
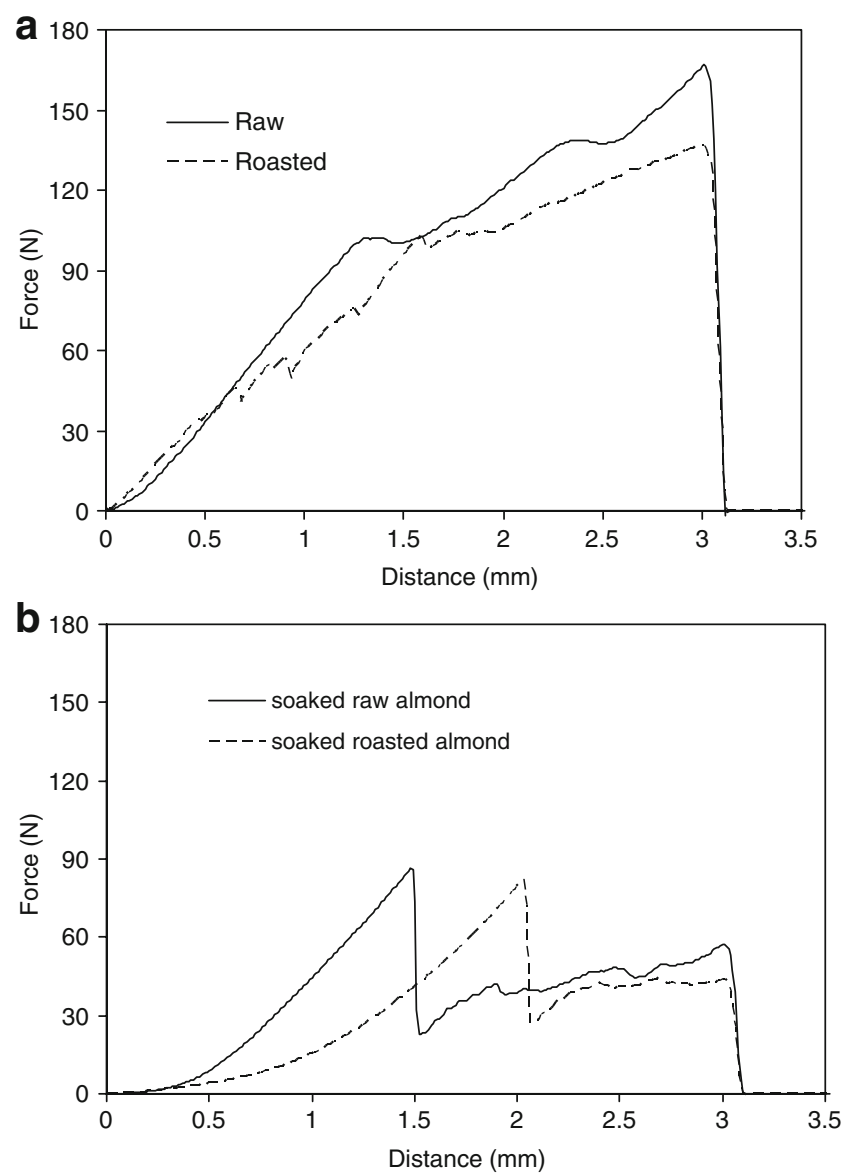

Fig. 4 Typical compression curves (force-distance [deformation] curve) for raw and roasted almonds before soaking in simulated gastric juice (a) and after soaking for $6.5 \mathrm{~h} \mathrm{(b)}$

profile of roasted almonds than the raw samples, indicating a more crispy texture for roasted almonds, as a result of reduced moisture content, increased porosity, and structural alteration during the roasting process. ${ }^{12}$ The average stiffness value for almonds before soaking slightly increased after roasting, although this difference is not significant (Table 3).

Absorption of gastric juice caused a significant decrease in hardness, stiffness, and total energy input due to increase in moisture and hydrolysis and enzymatic reactions that modified the structure (Table 3). Soaking caused significant differences between the texture of raw and roasted almonds. After soaking, raw almonds had a significantly higher stiffness $(67.23 \mathrm{~N} / \mathrm{mm})$ than roasted almonds $(49.68 \mathrm{~N} / \mathrm{mm}$; Table 3). The hardness and total work input in the raw almonds are also slightly higher. This might be related to the cellular and structural disruption that takes place during roasting resulting in a loss in elasticity of roasted almonds during water absorption. The lower stiffness values in the soaked roasted almonds than the raw almonds are in good agreement with the increase in their disintegration rate, 
Table 3 Textural parameters obtained with compression of a 50-mm flat-end probe for $3 \mathrm{~mm}$ depth on raw and roasted almond samples before and after soaking in simulated gastric juice for $6.5 \mathrm{~h}$ (sample size $=6 \times 6 \times 4 \mathrm{~mm} ; n=10$ )

\begin{tabular}{|c|c|c|c|c|c|}
\hline & $F(\mathrm{~N})$ & $\delta(\mathrm{mm})$ & $H(\mathrm{~N})$ & $A(\mathrm{~N} \mathrm{~mm})$ & $S(\mathrm{~N} / \mathrm{mm})$ \\
\hline \multicolumn{6}{|c|}{ Before soaking } \\
\hline Raw & $91.13 \pm 17.53 \mathrm{a}$ & $1.04 \pm 0.22 b$ & $177.91 \pm 14.34 \mathrm{a}$ & $293.25 \pm 14.95 \mathrm{a}$ & $88.29 \pm 7.47 \mathrm{a}$ \\
\hline Roasted & $64.39 \pm 28.03 b$ & $0.64 \pm 0.20 \mathrm{c}$ & $161.25 \pm 22.25 \mathrm{a}$ & $301.80 \pm 39.32 \mathrm{a}$ & $99.27 \pm 23.49 \mathrm{a}$ \\
\hline \multicolumn{6}{|c|}{ After soaking for $6.5 \mathrm{~h}$} \\
\hline Raw & $84.44 \pm 25.85 \mathrm{a}$ & $1.24 \pm 0.26 \mathrm{~b}$ & $66.09 \pm 8.52 b c$ & $125.61 \pm 16.16 \mathrm{~b}$ & $67.23 \pm 13.05 b$ \\
\hline Roasted & $92.05 \pm 22.52 \mathrm{a}$ & $1.85 \pm 0.33 \mathrm{a}$ & $58.57 \pm 18.65 \mathrm{c}$ & $112.49 \pm 25.55 b c$ & $49.68 \pm 8.64 c$ \\
\hline
\end{tabular}

$F$ fracture force, $\delta$ deformation at the fracture point, $H$ hardness at $3 \mathrm{~mm}$ depth, $A$ compression work, $S$ stiffness. Different letters within a column indicate significant differences $(p<0.05)$.

indicating that stiffness of soaked almonds may be a suitable indicator of the disintegration properties.

\section{Penetration Test}

The penetrometry profiles (force vs. depth of penetration) of raw and roasted almonds before and after soaking are shown in Figure 5a, b. These profiles show significantly different features. In Figure 5a, for the almonds before soaking, there is a sudden drop in the curves after first fracture, indicating that a break occurred in the sample due to the crisp nature of their texture, while this phenomenon did not occur for the soaked samples as the crispness decreased after absorbing water. Moreover, these profiles show that, following the first fracture point, the resistance may increase, decrease, or remain unchanged. According to Bourne ${ }^{13}$ force-distance curve obtained from penetration or puncture tests can be categorized into different types based on the direction of force change after fracture point (yield point). In type A, the force continues to increase after the yield point; in type B, the force is approximately constant after the yield point; and in type $\mathrm{C}$, the force decreases after the yield point. In Figure 5a, raw almond before soaking showed mostly type A and B curves while all of the roasted almond showed type A curve, which is related to a more crisp or brittle texture in the roasted almonds. After soaking, raw almonds showed type B curve while roasted almonds showed type $\mathrm{C}$ curve, indicating a "woody" texture in roasted almonds as a result of water absorption during soaking. ${ }^{13}$

Table 4 shows the mean values of different textural parameters. Before soaking, raw and roasted samples had no obvious trend in the changes of fracture force, hardness, stiffness, and the total work input. However, after soaking, except fracture force, all the parameters showed significant differences between the raw and roasted almonds, and the raw almonds had significantly higher values for all the parameters, which correspond to the slower disintegration of the raw almonds during digestion.
From Tables 3 and 4 and Figures 4 and 5, we can observe that both compression and penetration tests are able to characterize the significant difference between the rigidity of raw and roasted almonds after absorbing gastric juice, as indicated by the stiffness obtained from the
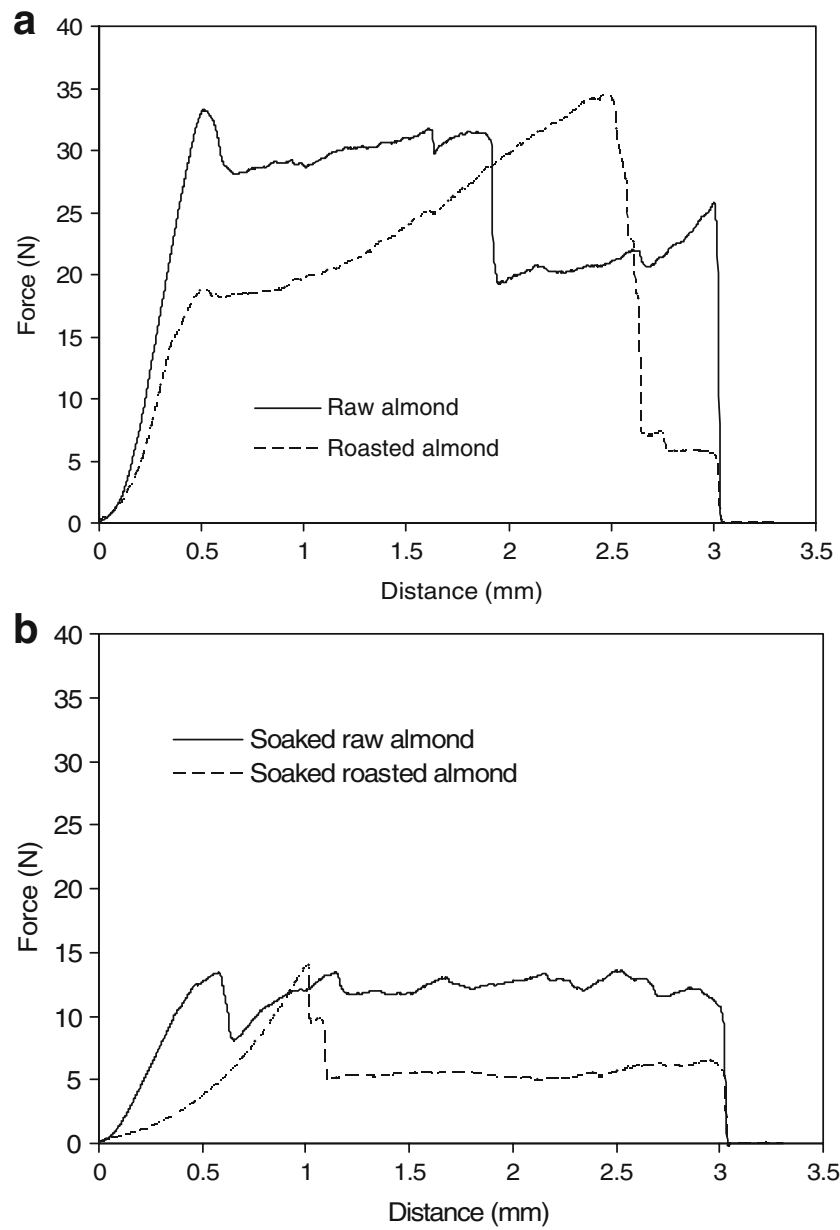

Fig. 5 Typical penetration curves (force-distance [deformation] curve) for raw and roasted almonds before soaking in simulated gastric juice (a) and after soaking for $16 \mathrm{~h}(\mathbf{b})$ 
Table 4 Textural parameters obtained with penetration of a 2-mm needle for $3 \mathrm{~mm}$ depth in raw and roasted almond samples before and after $16 \mathrm{~h}$ soaking in simulated gastric juice (sample size $=10 \times 10 \times 4 \mathrm{~mm} ; n=10$ )

\begin{tabular}{|c|c|c|c|c|c|}
\hline & $F(\mathrm{~N})$ & $\delta(\mathrm{mm})$ & $H(\mathrm{~N})$ & $A(\mathrm{~N} \mathrm{~mm})$ & $S(\mathrm{~N} / \mathrm{mm})$ \\
\hline \multicolumn{6}{|c|}{ Before soaking } \\
\hline Raw & $29.15 \pm 3.56 \mathrm{ab}$ & $0.52 \pm 0.06 \mathrm{a}$ & $29.34 \pm 2.80 \mathrm{a}$ & $77.06 \pm 29.12 \mathrm{a}$ & $56.36 \pm 9.21 \mathrm{a}$ \\
\hline Roasted & $21.68 \pm 3.00 \mathrm{~b}$ & $0.43 \pm 0.08 \mathrm{a}$ & $34.44 \pm 3.48 \mathrm{ab}$ & $96.00 \pm 14.84 \mathrm{ab}$ & $51.45 \pm 12.95 \mathrm{a}$ \\
\hline \multicolumn{6}{|c|}{ After soaking for $16 \mathrm{~h}$} \\
\hline Raw & $13.12 \pm 1.24 \mathrm{c}$ & $0.50 \pm 0.06 \mathrm{c}$ & $12.22 \pm 0.89 \mathrm{c}$ & $62.68 \pm 5.50 \mathrm{c}$ & $26.55 \pm 5.22 b$ \\
\hline Roasted & $13.32 \pm 1.30 \mathrm{c}$ & $1.02 \pm 0.11 \mathrm{~b}$ & $7.55 \pm 1.42 \mathrm{~d}$ & $36.26 \pm 4.36 \mathrm{~d}$ & $13.23 \pm 2.03 \mathrm{c}$ \\
\hline
\end{tabular}

$F$ fracture force, $\delta$ deformation at the fracture point, $H$ hardness at $3 \mathrm{~mm}$ depth, $A$ penetration work, $S$ stiffness. Different letters within a column indicate significant differences $(p<0.05)$.

compression and penetration tests. The lower rigidity of soaked roasted almonds than soaked raw almonds had a good correlation with the higher rate of disintegration in the roasted almonds, indicating that rigidity of soaked almonds might be a better tool to predict gastric disintegration rate of almonds. However, penetration test is more preferable as it can also differentiate hardness and total energy input during penetration between raw and roasted almonds after soaking. This may be due to the different principles involved in compression and penetration tests: in the compression test, only compression force is involved, whereas both compressive and shear forces are involved in the penetration tests. ${ }^{20}$

\section{Microstructure of Raw and Roasted Almonds During Digestion}

Mechanical and textural properties of a food reflect the structural arrangements of elements in the microstructure. ${ }^{21}$ The changes in the almond texture are dependent on alterations in the microstructure that take place during the roasting and digestion process. Figure $6 \mathrm{a}, \mathrm{b}$ shows the LM pictures of raw and roasted almonds after soaking in simulated gastric juice for $1 \mathrm{~h}$. The shaded area reflects a diffusion of resin into the tissue, while the white area indicates no resin diffusion. We observed that the shaded area of roasted almonds is significantly larger, i.e., the resin penetrated significantly deeper into the roasted sample tissue than raw almonds (Figure 6), which may be due to the looser structure of roasted almonds than raw almonds. In the edge area, broken cells and the empty cell walls can be seen where intracellular contents, such as lipid and protein bodies, were lost (Figures 6 and 7). It can be also seen that roasting created a significantly different structure. The shape of the cell changed and cell arrangement was extensively disrupted, as can be seen by comparing Figures $6 \mathrm{a}, 7 \mathrm{a}$ and $6 \mathrm{~b}, 7 \mathrm{~b}$. It has been reported that roasting causes disruption of the cytoplasmic network, denaturation and aggregation of protein bodies, coalescence of oil bodies, and increase in the volume of the intercellular space in almonds. ${ }^{4,5}$ The moisture decrease and parenchyma disruption is the principal cause of the increase in brittleness, crispness, and crunchiness in the roasted samples. $^{5}$

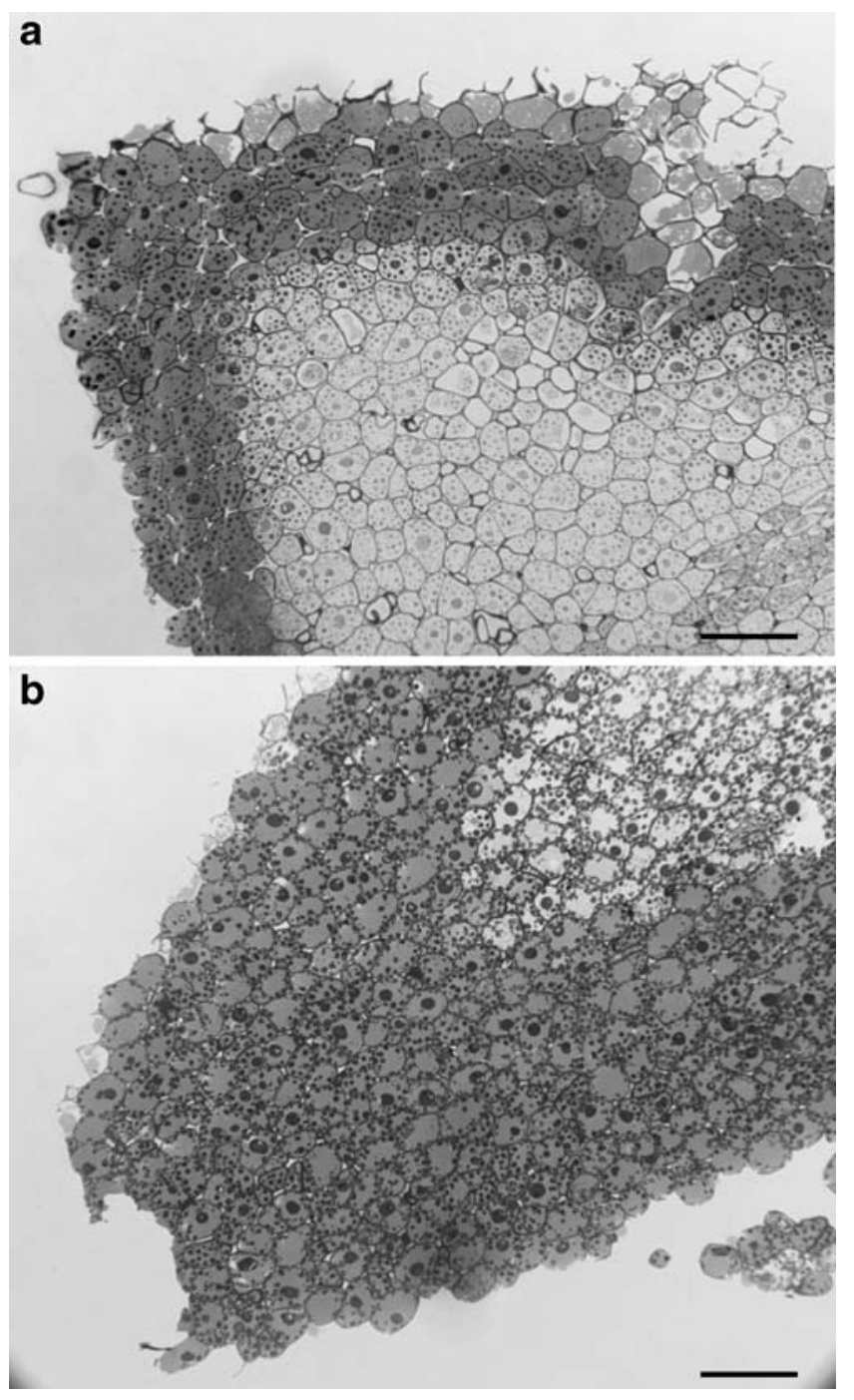

Fig. 6 LM images obtained after $1 \mathrm{~h}$ of static soaking in simulated gastric juice: a raw almond tissue, b roasted almond tissue. Bars indicate $100 \mu \mathrm{m}$ 
Fig. 7 LM images obtained after $1 \mathrm{~h}$ of static soaking in simulated gastric juice: a raw almond tissue, $\mathbf{b}$ roasted almond tissue. Bars indicate $50 \mu \mathrm{m}$

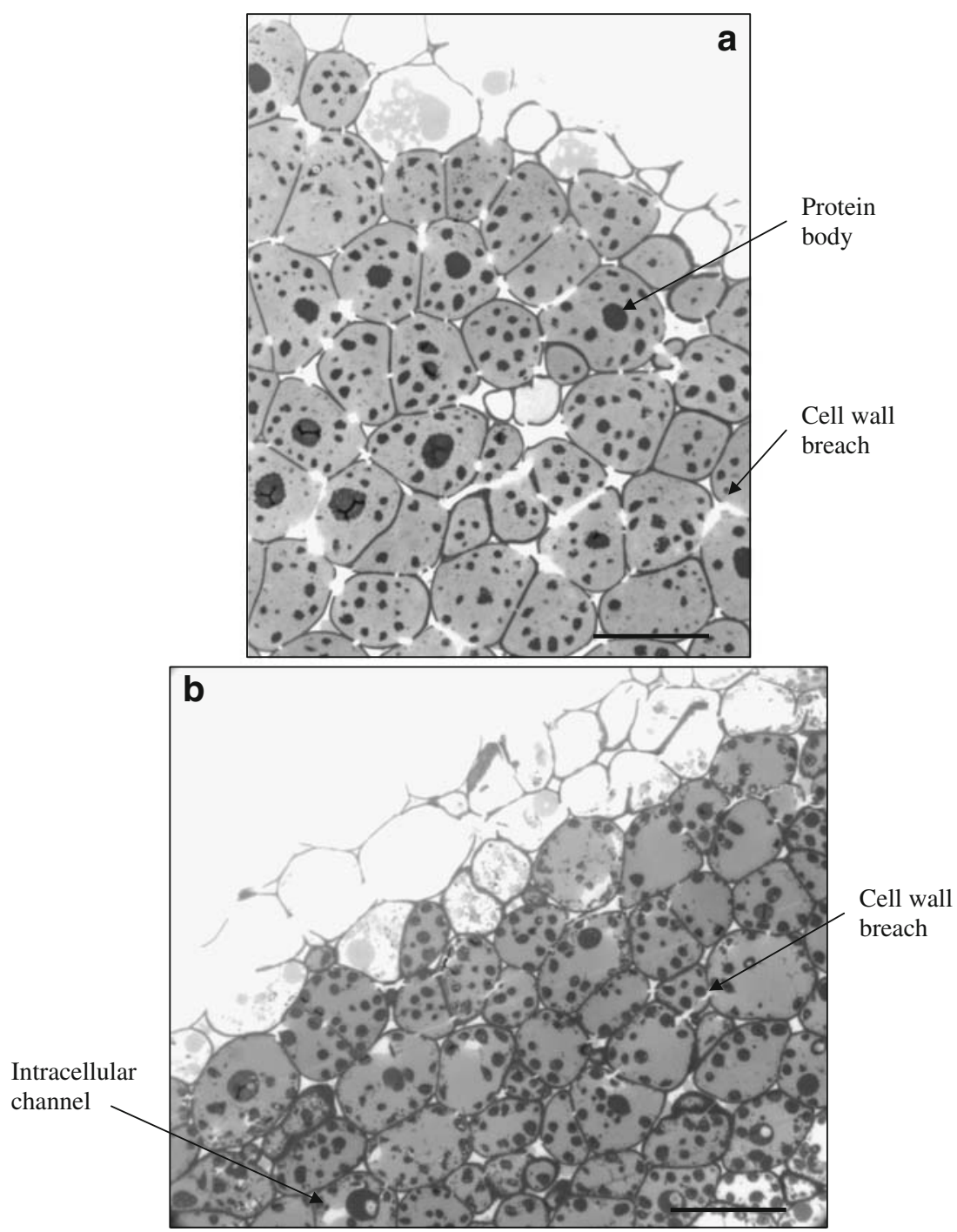

In Figure 7 (the shaded area), a result of the digestion effect can be clearly seen. Cell separation occurred due to the acidic hydrolysis of middle lamella reducing cell-cell adhesion; most cells in the fractured cell layer and some of the cells underlying fractured cell layer lost intracellular nutrients, showing only empty cell walls (Figure 7). Mandalari and others ${ }^{8}$ reported that the loss of intracellular nutrients from internal cells of raw almonds could only occur by a process of diffusion through the cell walls. However, we observed clear breach and breakage in the cell wall of most internal cells in the raw almond tissue, causing the intracellular nutrients to release from the breached locations (Figure 7a). We believe that the cell wall degradation resulting from breach and breakage may be a more important mechanism for the intracellular content to be digested, creating pathways through which the enzyme and acid gain access to the intracellular lipid and proteins bodies. For roasted almonds, we observed breach points not only along the cell walls but also in the intercellular spaces
(Figure 7b), as a result of heat treatment creating channels and voids caused by the cell shrinkage and escaping steam and oil. ${ }^{5}$ The disrupted cytoplasmic network and channels make up loose structure in the roasted almonds. Roasted almonds lost their elasticity/rigidity after absorption of moisture due to disrupted structure and high porosity developed during roasting. ${ }^{22}$

TEM micrographs provide a closer look on the changes in almond microstructure after roasting and digestion. The loss of cytoplasmic network, coalescence of oil drops, and aggregation of protein bodies can be clearly seen in the roasted almond cells by comparing Figure $8 \mathrm{a}, \mathrm{b}$. Loss of the middle lamella (pectin) and cell separation were evident and the cell wall degradation and breakage were clearly seen in both raw and roasted almonds facilitating nutrient release through the breached regions (Figure 8). This also caused a decrease in the rigidity and hardness of almonds after absorption of gastric juice as the mode of breakage changed from cell 
Fig. 8 TEM imaging obtained after $1 \mathrm{~h}$ of static soaking in simulated gastric juice: a raw almond tissue, $\mathbf{b}$ roasted almond tissue. Bars indicate $10 \mu \mathrm{m}$

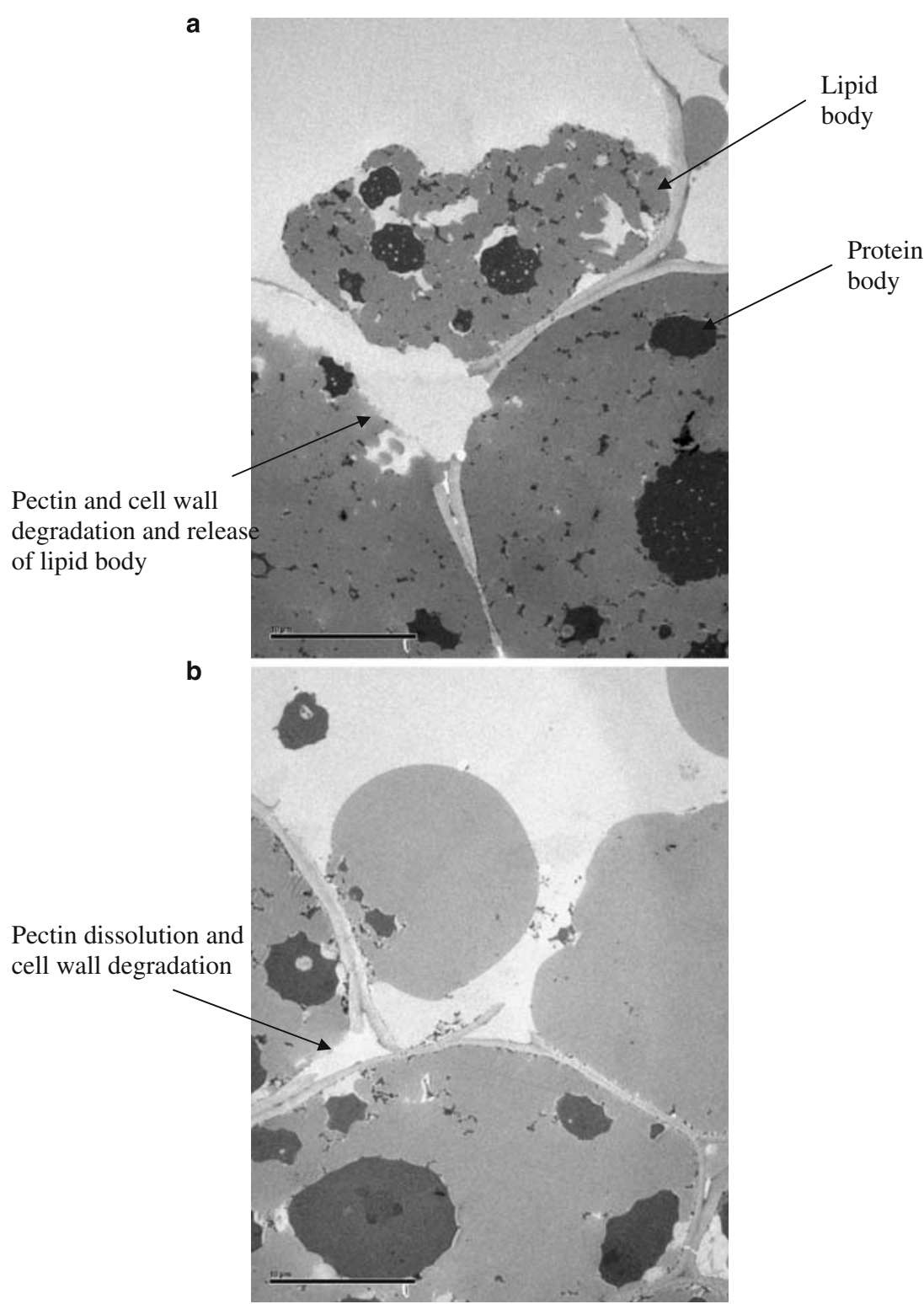

rupture to mostly cell separation when subjected to a compression force. $^{21}$

\section{Conclusion}

Compared with raw almonds, roasted almonds had less ability to absorb gastric juice and lower swelling capacity in simulated gastric environment. Reduced almond size led to a faster disintegration rate (shorter half time) and a higher level of swelling. The long half time of disintegration and the high amount of swelling of the almonds in the stomach may contribute to their high satiety property. While there was no significant difference in the solid loss (nutrient release) in static soaking and trial $\mathrm{A}$ in which only hydrodynamic mixing was involved, roasting significantly improved the disintegration rates of almonds and the solid loss when mechanical force was present. The internal resistance is more important than external resistance for leaching of nutrients from almond kernel to gastric environment. Both compression and penetration tests indicated that stiffness and rigidity of almonds after absorbing gastric juice was well correlated with the different disintegration rates of raw and roasted almonds. Examination of microstructure indicated that the breakage and breach of cell walls are important for almond digestion.

Acknowledgments This research was supported in part by USDA-NRI, contract 2009-35503-05195. Microscopy imaging was conducted at the Electron Microscopy Laboratory in the Department of Pathology and Laboratory Medicine, University of California, Davis. 
Open Access This article is distributed under the terms of the Creative Commons Attribution Noncommercial License which permits any noncommercial use, distribution, and reproduction in any medium, provided the original author(s) and source are credited.

\section{References}

1. US Department of Agriculture (USDA). ERS Food Availability (Per Capita) Data System, http://www.ers.usda.gov/data/ foodconsumption/. Accessed 21 November 2008

2. C.Y. Chen, K. Lapsley, J. Blumberg, A nutrition and health perspective on almonds. J. Sci. Food Agric. 86, 2245-2250 (2006)

3. P.P. Hoppe, G. Krennrich, Bioavailability and potency of natural source and all-racemic R-tocopherol in the human: a dispute. Eur. J. Nutr. 39, 183-193 (2000)

4. A. Kita, A. Figiel, The effect of roasting on the texture of walnuts. Acta Agrophys. 7(1), 87-97 (2006)

5. P. Varela, J.M. Aguilera, S. Fiszman, Quantification of fracture properties and microstructural features of roasted marcona almonds by image analysis. Lebensm.-Wiss. Technol. 41, 10-17 (2008)

6. P.R. Ellis, C.W.C. Kendall, Y. Ren, C. Parker, J.F. Pacy, K.W. Waldron, D.J.A. Jenkins, Role of cell walls in the bioaccessibility of lipids in almond kernels. Am. J. Clin. Nutr. 80, 604-613 (2004)

7. F. Kong, R.P. Singh, A model stomach system to investigate disintegration kinetics of solid foods during gastric digestion. J. Food Sci. 73(5), E202-E210 (2008)

8. G.R.M. Mandalari, G.T. Faulks, R.V. Lo, D.R. Turco, R.B. Picout, G. Lo Curto, P.D. Bisignano, G. Dugo, K.W. Waldron, P.R. Ellis, M.S.J. Wickham, Release of protein, lipid and vitamin E from almond seeds during digestion. J. Agric. Food Chem. 56, 3409-3416 (2008)

9. F. Kong, R.P. Singh, Disintegration of solid foods in human stomach (review). J. Food Sci. 73(5), R67-R80 (2008)

10. F. Kong, R.P. Singh, Modes of disintegration of solid foods in simulated gastric environment. Food Biophys. 4, 180-190 (2009)
11. A. Borges, M. Peleg, Effect of water activity on the mechanical properties of selected legumes and nuts. J. Sci. Food Agric. 75, 463-471 (1997)

12. P. Pittia, M. Dalla Rosa, C.R. Lericis, Textural changes of coffee beans as affected by roasting conditions. Lebensm.-Wiss. Technol. 34, 168-175 (2001)

13. M. Bourne, Food Texture and Viscosity: Concept and Measurement, 2nd edn. (Academic, San Diego, 2002)

14. A. Pal, K. Indireshkumar, W. Schwizer, B. Abrahamsson, M. Fried, J.G. Brasseur, Gastric flow and mixing studied using computer simulation. Proc. R. Soc. Lond. B Biol. Sci. 271, 25872594 (2004)

15. M.A. Peyron, A. Mishellany, A. Woda, Particle size distribution of food boluses after mastication of six natural foods. J. Dent. Res. 83(7), 578-582 (2004)

16. J.M. Frecka, J.H. Hollis, R.D. Mattes, Effects of appetite, BMI, food form and flavor on mastication: almonds as a test food. Eur. J. Clin. Nutr. 61(7), 1-8 (2007)

17. J.B. Dressman, Comparison of canine and human gastrointestinal physiology. Pharm. Res. 3(3), 123-131 (1986)

18. R.D. Mattes, P.M. Kris-Etherton, G.D. Foster, Impact of peanuts and tree nuts on body weight and healthy weight loss in adults. J. Nutr. 138, 1741S-1745S (2008)

19. Y. Malkki, E. Virtanen, Gastrointestinal effects of oat bran and oat gum. A review. Lebensm.-Wiss. Technol. 34, 337-347 (2001)

20. M.C. Bourne, Measure of shear and compression components of puncture tests. Food Sci. 31, 282-291 (1966)

21. J.M. Aguilera, P.J. Lillford. Structure-property relationships in foods. In: Aguilera JM, Lillford PJ, editors. Food Materials Science: Principles and Practice, p. 229-253 (Springer Publisher, New York, 2007)

22. M.K. Krokida, C. Philippopoulos, Rehydration of dehydrated foods. Dry. Technol. 23(4), 799-830 (2005)

23. S. Aoki, K. Uesugi, K. Tatsuishi, H. Ozawa, M. Kayano, Evaluation of the correlation between in vivo and in vitro release of phenylpropanolamine $\mathrm{HCl}$ from controlled-release tablets. Int. J. Pharm. Med. 85, 65-73 (1992) 